\title{
Myxoid Meningioma: An Example of a Rare Brain Tumour of Difficult Diagnosis
}

\author{
Javier Ortiz $^{1 *}$, María Dolores Ludeña ${ }^{1}$, Jesús Gonçalves ${ }^{2}$, Sofía Del Carmen ${ }^{1}$, Ángel Maillo ${ }^{2}$, \\ Agustín Bullon ${ }^{1}$ \\ ${ }^{1}$ Pathology Department, Hospital Universitario de Salamanca, (Complejo Hospitalario de Salamanca), Salamanca, Spain; ${ }^{2}$ Neuro- \\ surgery Department, Hospital Virgen de la Vega, (Complejo Hospitalario de Salamanca), Salamanca, Spain. \\ Email: *jortiz@usal.es
}

Received October $22^{\text {nd }}, 2012$; revised November $23^{\text {rd }}, 2012$; accepted December $13^{\text {th }}, 2012$

\begin{abstract}
We present a case of a Myxoid Meningioma noticed in a 43-year-old woman. The patient, who had no noteworthy medical antecedents, showed repeated focal epileptic crisis. Imaging studies (MR and CT) showed a solitary and well delimited tumour located in the frontral lobe. Once extirpated, the histological study revealed a neoplasm of myxoid and benign appearance, with elongated and uniform cells, with no atypias and a very low mitotic index. Immunohistochemisty studies (positivity for Vimentin and Epithelial Membrane Antigen, and negativity for the other markers) as well as physical and radiological examination of the patient (absence of tumours in any other location), suggested the diagnosis of a Myxoid Meningioma, which was confirmed by electron microscopy (by the presence of desmosomes). We also revise the characteristics of Myxoid Meningiomas, an absolutely exceptional primary tumour of the central nervous system.
\end{abstract}

Keywords: Myxoid Meningioma; Immunohistochemistry; Electron Microscopy

\section{Introduction}

The spectacular advance in the comprehension of primary tumor genetic alterations, has made possible the use of more efficient and more specific antitumor treatments. These therapies, with their secondary effects, have obliged practitioners, radiologists and pathologists to submit more accurate diagnosis and therefore, to know in depth and exhaustively about the different neoplasms, not only the more frequent ones, but also those uncommon and difficult to notice in the daily practice.

This logical statement, at least for us, acquires a special significance when we face up to cases like this, absolutely infrequent

\section{Clinical Case}

A 43-year-old woman, with any interesting medical antecedent, who came to our hospital because of repeated focal epileptic crisis. Radiological studies (MR and TC) showed a solitary and well delimited tumour of $1 \mathrm{~cm}$ of diameter located in the frontal lobe (Figure 1).

The well demarcation of the tumour, allowed to its surgical enucleation, observing an encapsulated and gelatinous lesion. The histological study (Figure 2(a)) re-

${ }^{*}$ Corresponding author. vealed a markedly myxoid neoplasm consisting of polygonal and fusiform cells with no evident cytological atypias and with a very low mitotic index (lower than 1 mitoses per 50 High Power Fields).

In the immunohistochemical studies, tumour cells showed positivity for Vimentin and EMA (Figure 2(b)), and negativity for Cytokeratins (AE1/AE3 and CAM 5.2), HMB.45, S-100, Actin, Desmin, CD117, Neurofilaments and GFAP. Progesterone receptors were also negative and CD31 and CD34 were positive only in vessels. The proliferation activity marker (MIB1), was lower than $1 \%$.

Complementary studies with electron microscopy revealed the presence of intercellular desmosomal junctions, interdigitations and microtubules (Figure 3).

Radiological complementary studies (TC and heart ultrasonography) dismissed the presence of any other tumour out of the central nervous system.

On the basis of all these findings, the tumour was diagnosed as a Myxoid Meningioma (grade I Meningioma), primary from the central nervous system.

\section{Discussion}

The 2007 WHO classification [1], distinguishes three different types of Meningiomas according to their biological behaviour (grade I, II and II Meningiomas), in- 


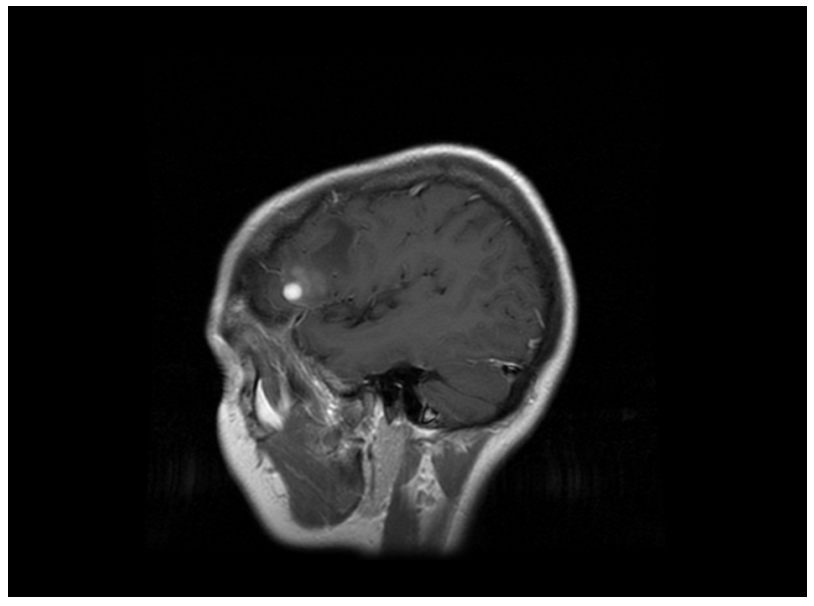

Figure 1. MRI: image of a well delimited lession located in frontal lobe.

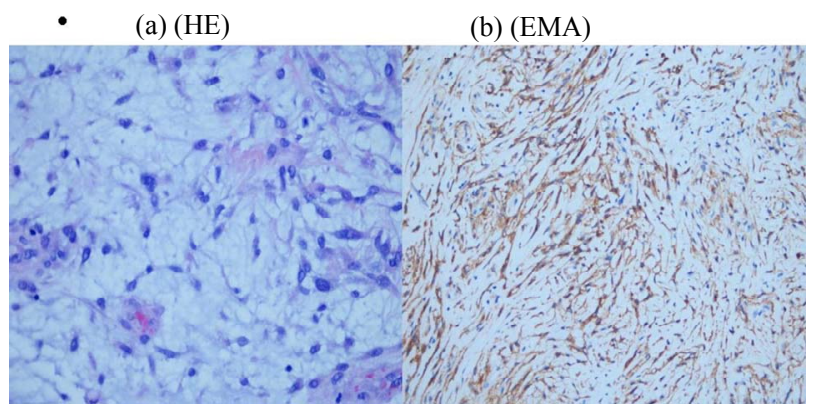

Figure 2. (a) Myxoid Meningioma: Myxoid tumour with scattered cells of benign appearance $(\mathrm{He} \times 200)$; (b) Myxoid Meningioma: Tumour cells showed positivity for EMA (I h $\times 200)$.
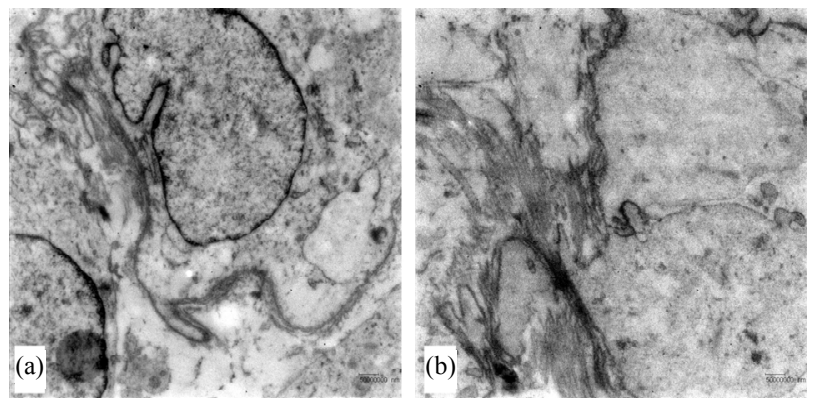

Figure 3. Myxoid Meningioma: (ultra structure). (a) Presence of interdigitations between tumour cells (Transmission Electron Microscopy); (b) Presence of intercellular unions and microtubules (Transmission Electron Microscopy).

cluding a wide range of histological variants in the less aggressive group (grade I Meningiomas). One of these variants is Metaplastic meningiomas [2-4], which are those that show areas constituted of non meningothelial mature tissues such as cartilage (Chondroid Meningioma), adipose tissue (Lipomatous Meningioma), foamy histiocytes (Xantomatous Meningioma) or bone tissue
(Ossificant Meningioma). It is in this curious section of Metaplastic meningiomas where the Myxoid Meningioma is included. Myxoid Meningioma is an infrequent variant and difficult to diagnose, and as its name indicates, this tumour is characterized by a marked myxoid transformation and a very different histological appearance if compared to usual Meningiomas. All this makes this lesion subject to be mistaken with other tumours of different histological linage and with a very different prognosis [5-7].

In our case we found an example of a Myxoid Meningioma, which needed several studies to differentiate it from other entities. Immunohistochemistry confirmed positivity for Vimentin and EMA, and this allowed us to recognize a profile that suggested a meningothelial tumour. Negative stain for other markers, also allowed us to reject other possibilities such a metastases from a mucinous tumour (positivity for Cytokeratins), Cranial Chordoma or Ecchordosis physaliphora (Cytokeratin and S-100 positive), Choroid glioma from the central nervous system (GFAP positive), Neurinoma (S-100 positive), Neurofibroma (S-100, CD-34 and Neurofilaments positive), Neurotecoma (S-100, GFAP positive), Melanomas, either primary or metastatic (HMB-45 positive), Myxoid Leiomyosarcoma (Actin positive), Rhabdomyoma and Rhabdomyosarcoma (Actin and Desmin positive), Myxoid Chondrosarcoma (S-100 positive) and a GIST metastasis (CD 117 positive) [8-12]. Radiological studies ratified the results obtained by the immohistochemical analysis, rejecting the possibility of a malignant metastatic process, or the embolism of a Cardiac Myxoma [13]. Electron microscopy revealed the existence of intercellular unions, and definitively confirmed the meningothelial origin of the neoplasm [14].

Among all Meningiomas, it also should be made a differential diagnosis with Chordoid Meningioma (grade I Meningioma), which was easily rejected because no typical cytological images were found (similar in some degree to those in the Chordoma) $[15,16]$.

In our opinion, the presentation of this case, can serve to release a rare neoplasm with a difficult diagnose, as it has been proved, as well as to reaffirm the importance of the collaboration between practitioners, radiologists and pathologist, which is the best for a right clinical practice.

\section{REFERENCES}

[1] D. N. Louis, H. Ohgaki, O. D. Wiestler, W. K. Cavenee, P. C. Burger, A. Jouvet, B. W. Scheithauer and P. Kleihues, "The 2007 WHO Classification of Tumours of the Central Nervous System," Acta Neuropathologica, Vol. 114, No. 2, 2007, pp. 97-109.

[2] R. Ijiri, M. Hara, Y. Tanaka, K. Kato and K. Sekido, "Spinal Cartilaginous Meningioma in a Child: A Case Report," Child's Nervous System, Vol. 16, No. 2, 2000, 
pp. 80-83. doi:10.1007/PL00007284

[3] H. Ikota and Y. Nakazato, "A Case of Metaplastic Meningioma with Extensive Xanthomatous Change," Neuropathology, Vol. 28, No. 4, 2008, pp. 422-426. doi:10.1111/j.1440-1789.2008.00865.x

[4] V. Barresi, M. Caffo, A. Ieni, C. Alafaci and G. Tuccari, "Osteoblastic Meningiomas: Clinico-Pathological and Immunohistochemical Features of an Uncommon Variant," Journal of Neuro-Oncology, Vol. 105, No. 2, 2011, pp. 225-232. doi:10.1007/s11060-011-0588-3

[5] J. D. Harrison and P. E. Rose, "Myxoid Meningioma: Histochemistry and Electron Microscopy," Acta Neuropathologica, Vol. 68, No. 1, 1985, pp. 80-82. doi:10.1007/BF00688961

[6] L. R. Bégin, "Myxoid Meningioma," Ultrastructural Pathology, Vol. 14, No. 4, 1990, pp. 367-374. doi:10.3109/01913129009032251

[7] K. M. Krisht, T. Altay and W. T. Couldwell, "Myxoid Meningioma: A Rare Metaplastic Meningioma Variant in a Patient Presenting with Intratumoral Hemorrhage," Journal of Neurosurgery, Vol. 116, No. 4, 2012, pp. 861-865. doi:10.3171/2011.12.JNS111020

[8] H. Y. Cho, M. Lee, H. Takei, J. Dancer, J. Y. Ro and Q. J. Zhai, "Immunohistochemical Comparison of Chordoma with Chondrosarcoma, Myxopapillary Ependymoma, and Chordoid Meningioma," Applied Immunohistochemistry \& Molecular Morphology, Vol. 17, No. 2, 2009, pp. 131138. doi:10.1097/PAI.0b013e3181866a13

[9] S. S. Ling, C. Sader, P. Robbins and G. P. Rajan, "A Case of Giant Ecchordosis Physaliphora: A Case Report and Literature Review," Otology \& Neurotology, Vol. 28, No. 7, 2007, pp. 931-933.
[10] B. Can, "Cytology of Chordoid Glioma of the Third Ventricle," Diagnostic Cytopathology, Vol. 40, No. 2, 2012, pp. 185-187. doi:10.1002/dc.21619

[11] A. Mekni, E. Braham, K. Nouira, H. K. Ben, K. Bellil, S. Haouet, N. Kchir, M. Khaldi and M. Zitouna, "Primary Central Nervous System Malignant Melanoma: Report of 5 Cases," Pathologica, Vol. 99, No. 3, 2007, pp. 71-75.

[12] N. A. Wong, "Gastrointestinal Stromal Tumours-An Update for Histopathologists," Histopathology, Vol. 59, No. 5, 2011, pp. 807-821. doi:10.1111/j.1365-2559.2011.03812.x

[13] N. Kohno, Y. Kawakami, C. Hamada, G. Toyoda, H. Bokura and S. Yamaguchi, "Cerebral Embolism Associated with Left Atrial Myxoma That Was Treated with Thrombolytic Therapy," Case Reports in Neurology, Vol. 4, No. 1, 2012, pp. 38-42.

[14] K. Yamazaki and B. Eyden, "An Ultrastructural and Immunohistochemical Study of Microcystic Meningioma with Emphasis on Matrix Proteins and Connexin 26 Type Gap Junctions," Ultrastructural Pathology, Vol. 28, No. 4, 2004, pp. 247-253. doi:10.1080/019131290505257

[15] J. L. Wilson, T. L. Ellis and R. T. Mott, "Chordoid Meningioma of the Third Ventricle: A Case Report and Review of the Literature," Clinical Neuropathology, Vol. 30, No. 2, 2011, pp. 70-74.

[16] S. L. Zhao, Y. Li, X. Y. Tian, Z. Li, Q. Huang and B. Li, "Intraparenchymal Cystic Chordoid Meningioma: A Case Report and Review of the Literature," Neuropathology, Vol. 31, No. 6, 2011, pp. 648-653. doi:10.1111/j.1440-1789.2011.01214.x 